\title{
348 - Increased White Matter Hyperintensity and Brain Resting-state fMRI Topology Changes in Suicide Attempters of Late-life Depression
}

Chemin, Lin ${ }^{\mathrm{a}, \mathrm{b}, \mathrm{c}}$ Chih-Mao, Huang ${ }^{\mathrm{d}, \mathrm{e}}$ Ho-Ling Anthony, Liu ${ }^{f}$ Changwei W., Wu ${ }^{\mathrm{g}, \mathrm{h}} \quad$ Cheng Hong, Toh ${ }^{\mathrm{b}, \mathrm{l}}$ Yun-Fang Tsai ${ }^{a, j, k}$ Tatia Mei-Chun, Lee ${ }^{1, m}$ Shwu-Hua, Lee ${ }^{b, n *}$

a. Department of Psychiatry, Keelung Chang Gung Memorial Hospital, Keelung, Taiwan

b. College of Medicine, Chang Gung University, Taoyuan County, Taiwan.

c. Community Medicine Research Center, Chang Gung Memorial Hospital, Keelung, Taiwan

d. Department of Biological Science and Technology, National Chiao Tung University, Hsinchu, Taiwan

e. Cognitive Neuroscience Laboratory, Institute of Linguistics, Academia Sinica, Taipei, Taiwan

f. Department of Imaging Physics, University of Texas MD Anderson Cancer Center, Houston, Texas

g. Brain and Consciousness Research Center, Shuang-Ho Hospital, New Taipei, Taiwan

h. Graduate Institute of Mind, Brain and Consciousness, Taipei Medical University, Taipei, Taiwan

i. Department of Medical Imaging and Intervention, Chang Gung Memorial Hospital at Linkou, Taoyuan County, Taiwan

j. School of Nursing, College of Medicine, Chang Gung University, Tao-Yuan City, Taiwan

k. Department of Nursing, Chang Gung University of Science and Technology, Tao-Yuan City, Taiwan

1. Laboratory of Neuropsychology, The University of Hong Kong, Hong Kong

m. State Key Laboratory of Brain and Cognitive Science, The University of Hong Kong, Hong Kong

n. Department of Psychiatry, Linkou Chang Gung Memorial Hospital, Taoyuan County, Taiwan

* Corresponding author; Email: shlee@cgmh.org.tw

Supported by (1) medical research grants CMRPG3C0041/42 from Chang Gung Memorial Hospital and NRRPG2H0031 from Ministry of Science and Technology, Taiwan to Chemin Lin (2) NMRPG3G6031/32 from Ministry of Science and Technology, Taiwan to Shwu-Hua, Lee (3) the KKHo International Charitable Foundation to Tatia Lee.

Introduction: Suicide rate tends to peak in old age, and major depression is the most salient risk factor for late-life suicide. However, few studies have focused on the neuroscientific facet of suicide in the context of late-life depression (LLD).

Methods: We recruited 114 participants of LLD ( 28 with history of suicide attempt and 86 without) and 47 elderly controls. They received MRI scanning and behavioral assessment. White matter hyperintensity $(\mathrm{WMH})$ was quantified by an automated segmentation algorithm and graph theoretical analysis was applied to resting-state fMRI. We used ANCOVA to compare group difference in WMH loading and multivariate generalized linear model to compare global and local topological parameters in fMRI signals, controlling for demographics. Partial correlation was conducted between imaging parameters and behavioral data in group of suicide attempters.

Results: We found significant higher WMH in suicide attempters than those of LLD without suicide attempts and elderly controls $(F=7.091 ; p=0.001)$. Suicide attempters also had increased betweenness centrality $(B C)$ in right superior occipital gyrus (SOG) (Bonferroni corrected), right precuneus (False positive corrected) and right superior temporal gyrus (uncorrected) and decreased BC in left hippocampus (uncorrected). In suicide attempters, higher BC in right SOG correlated with higher WMH, higher depression severity, higher illness awareness and insight, and lower cognitive function (digit backward), while higher $\mathrm{BC}$ in right precuneus correlated with higher decrease awareness and insight and higher cognitive function (digit backward).

Conclusion: Resonating with the vascular hypothesis in LLD, higher WMH was found in those having history of suicide attempts. However, the re-organized brain topology changes are related with divergent cognitive function and convergent heightened disease insight. 
Key words: suicide; white matter hyperintensity; late-life depression; cognitive function ; insight ; graph theory 